\title{
Marcello Cesa-Bianchi e gli sviluppi della psicologia italiana dagli anni '50 al 2000
}

\section{Marcello Cesa-Bianchi and the development of Italian psychology from the 1950s to 2000}

\author{
Mauro Antonelli*, Alessandro Porro**, Carlo Cristini^ ${ }^{\wedge}$ \\ *Dipartimento di Psicologia, Università degli Studi di Milano-Bicocca, \\ Piazza dell'Ateneo Nuovo, 1, 20126 Milano, tel. 0264483730 \\ e-mail: mauro.antonelli@unimib.it \\ **Dipartimento di Scienze Cliniche e di Comunità, \\ Università degli Studi di Milano \\ Via Francesco Sforza, 35, 20122 Milano \\ e-mail: alessandro.porro1@unimi.it; tel: 0250320126 \\ ${ }^{\wedge}$ Dipartimento di Scienze Cliniche e Sperimentali, \\ Università degli Studi di Brescia \\ Viale Europa, 11, 25123 Brescia \\ e-mail: carlo.cristini@unibs.it; tel: 0303717489
}

Ricevuto: 10.04.2020 - Accettato: 07.07.2020

Pubblicato online: 07.05 .2021

\section{Riassunto}

Vasta è stata la produzione scientifica del Professor Marcello Cesa-Bianchi. Nella sua esperienza accademica, durata quasi settant'anni, ha trattato i più svariati temi della psicologia.

Nei primi anni di attività si è occupato soprattutto dell'invecchiamento, di metodologia sperimentale, di psicometria, di percezione. Successivamente numerosi altri argomenti sono stati oggetto di approfondimento. Dell'invecchiamento in particolare si è sempre interessato fino al termine della sua lunga vita accademica e scientifica.

In questo contributo di prospettiva storica vengono sinteticamente esaminate oltre ai lavori relativi alla metodologia, alla psicometria e alla percezione - le principali pubblicazioni del Professore riguardo agli studi e alle ricerche sulla psi-

M. Antonelli et al. / Ricerche di Psicologia, 2021, Vol. 44(1),

ISSN 0391-6081, ISSNe 1972-5620, Doi: 10.3280/rip1-2021oa11585

Copyright $\odot$ FrancoAngeli

This work is released under Creative Commons Attribution - Non-Commercial No Derivatives License. For terms and conditions of usage please see: http://creativecommons.org 
cologia dell'età evolutiva, sulla psicologia del lavoro, sulla psicologia medica, con un particolare riferimento alla formazione complessiva del medico, quali temi fra i più rappresentativi della sua imponente attività scientifica.

Parole chiave: Marcello Cesa-Bianchi, storia della psicologia, metodologia, percezione, età evolutiva, lavoro, medicina.

\begin{abstract}
Professor Marcello Cesa-Bianchi produced a vast body of scientific work. In his almost seventy years of academic experience he studied a wide range of psychology topics.

In his early working years, he mainly dealt with ageing, experimental methodology, psychometrics, and perception. Subsequently many other topics were the subject of deeper study. He was always particularly interested in ageing, until the end of his long academic and scientific life.

This contribution, from a historical perspective, summarises and examines in addition to the work relating to methodology, psychometrics and perception the Professor's main publications on his studies and research on developmental psychology, on psychology and on medical psychology with a particular reference to the overall training of the doctor, as some of the most representative themes of his impressive scientific work.
\end{abstract}

Keywords: Marcello Cesa-Bianchi, history, psychology, methodology, perception, childhood, work, medicine.

\title{
Premessa
}

Non vi è tema della psicologia che il Professor Marcello Cesa-Bianchi non abbia affrontato nella sua lunga vita accademica e scientifica, attestata da un elevato numero di pubblicazioni (Cristini e Porro, 2017).

Uno dei primi argomenti studiati (che qui trattiamo in estrema sintesi, giacché il tema sarà oggetto di un altro contributo nel presente volume), che potrebbe in qualche modo essere considerato un fil rouge che scorre lungo l'intera vita scientifica del Professore, è la psicogerontologia. Negli ultimi decenni della sua fecondissima attività, egli si dedicò in modo particolare a quest'ambito disciplinare con una serie di contributi in continua evoluzione. Si pensi alla valorizzazione della creatività (Cesa-Bianchi, 1998) e all'enucleazione di una sua particolare caratteristica temporale e qualitativa, definita ultima creatività (Cristini et al., 2011). I suoi apporti in argomento sono così numerosi che non possono essere qui compendiati, 
ma l'alfa e l'omega di questa sua produzione scientifica meritano di essere ricordati, perché corrispondono, in verità, all'alfa e all'omega della sua vita accademica e scientifica.

Indirizzato agli studi di psicologia dell'invecchiamento da padre $\mathrm{Ge}$ melli, il primo lavoro scientifico di Marcello Cesa-Bianchi (Cesa-Bianchi, 1951) fu dedicato all'applicazione dei reattivi mentali in persone anziane. Il viraggio verso una posizione autenticamente psicogerontologica fu rapido, contemporaneo all'uso critico dei reattivi mentali (per usare la terminologia dell'epoca). Nel decennio successivo era ormai emersa in tutta la sua rilevanza la necessità di una formalizzazione della metodologia di ricerca in campo psicogerontologico (Cesa-Bianchi, 1965a).

Due altri temi occupano, accanto alla psicologia dell'invecchiamento, l'interesse del giovane Cesa-Bianchi, verso i quali, anche in questo caso, era stato indirizzato dal maestro Gemelli: la metodologia (con la psicometria) e la percezione.

\section{Metodologia e psicometria}

Al problema metodologico Cesa-Bianchi ha sin dall'inizio riconosciuto un ruolo centrale data l'adesione a quel metodo sperimentale che allinea la psicologia alle altre scienze biologiche, per il quale avverte d'altra parte la necessità di adattare le procedure alla peculiarità dei processi psicologici, integrati ma distinguibili dai processi biologici dell'organismo.

L'adesione al metodo sperimentale lo induce ad approfondire gli strumenti più aggiornati introdotti nella sperimentazione psicologica, a partire dai modelli offerti dalla statistica, all'epoca ancora scarsamente diffusi in Italia. Esce così nel 1952, in collaborazione con Angelo Perugia, altro allievo di Gemelli, il primo libro di Marcello Cesa-Bianchi (Cesa-Bianchi e Perugia, 1952a).

Il volume offre una panoramica sulle prospettive, ma anche sui limiti, che caratterizzano la statistica in relazione a una disciplina particolare come la psicologia, in termini di devianza, indici di significatività, correlazioni ed elementi probabilistici. Cesa-Bianchi ritornava su questi temi nella prolusione (Cesa-Bianchi, 1966a), tenuta il 21 gennaio 1966, che inaugurava l'attività conseguente alla stipula della convenzione fra Comune e Università degli Studi di Milano e che rendeva concreta e stabile la collaborazione fra le due istituzioni.

Il tema scelto era proprio quello della metodologia, applicata alla psicologia quale campo particolare di azione. Se per la psicologia sperimentale il conflitto fra approccio qualitativo e quantitativo (determinato dalla statistica) poteva essere composto, allorché si entrava nel dato clinico (e nel suo metodo), le cose si complicavano. Andava ricercata la via media- 
na, anche se la distanza fra le due posizioni era ben più grande di quanto si potesse immaginare. Bastava, citando Paul Everett Meehl (1920-2003) (Meehl, 1954), rammentare gli appellativi che i fautori del metodo clinico attribuivano agli statistici e viceversa: si trattava di un florilegio di epiteti. In realtà, $i$ due metodi dovevano al contrario valorizzare $i$ propri dati (Cesa-Bianchi, 1966a) in un contesto di rigore, interdisciplinarità e verifica.

Anche in questo contesto la situazione della Facoltà di Medicina e Chirurgia dell'Università degli Studi di Milano si dimostrava lungimirante: si deve infatti segnalare l'istituzione di una cattedra di Statistica medica e biometria, per non parlare del testo di Enrico Poli (1909-1997) sulla Metodologia medica (Poli, 1965), che a oltre un sessantennio dalla sua comparsa non perde il valore di classico della disciplina.

Nel tempo non calò l'interesse per il problema metodologico, stante anche la sua valenza epistemologica e storica (Cavagna et al., 2017), e Marcello Cesa-Bianchi definì ulteriormente le due posizioni in gioco come statistico-probabilistica la prima e clinico-sociale la seconda, con un ampliamento semantico degno di nota (Cesa-Bianchi, 1987).

In campo strettamente psicometrico Cesa-Bianchi ha contribuito a superare lo scontro tra coloro che ritenevano il test uno strumento fondamentale, capace di quantificare le diverse funzioni e dimensioni mentali, e coloro che lo consideravano una procedura che altera, categorizzando e schematizzando, gli aspetti qualitativi della vita psichica.

Attraverso una serie di articoli, Cesa-Bianchi ha messo a punto vari test, sia di efficienza che di personalità, dalle scale ai questionari, proponendo da un lato nuovi strumenti, e traducendo e adattando dall'altro alla popolazione italiana reattivi elaborati in altri paesi, a partire dalla Scala Wechsler-Bellevue per la valutazione dell'intelligenza (Cesa-Bianchi e Perugia, 1952b). Le applicazioni hanno riguardato sia campioni di popolazione in diverse fasi del ciclo di vita, sia campioni clinici di pazienti neuropsichiatrici.

L'esito emergente da queste ricerche è da un lato l'indubbia utilità dei test a fini conoscitivi e diagnostici, ma, al tempo stesso, il loro valore non inequivocabile, che deve essere corroborato attraverso il confronto con i risultati ottenuti in altri test, nel quadro dell'applicazione di una batteria, la convalida attraverso criteri esterni, come la valutazione scolastica o clinica, o il loro utilizzo come convalida di ipotesi precedentemente formulate. 


\section{Percezione}

Un secondo filone di ricerca sviluppato dal giovane Cesa-Bianchi è stata la psicologia della percezione, che gli ha consentito di affrontare, in forma esemplare, alcuni aspetti essenziali dell'intera psicologia generale, dalle sue origini storiche ai suoi fondamenti logico-metodologici ed epistemologici.

In quest'ambito, all'epoca (siamo negli anni '50), si contrapponevano due scuole, quella gestaltista, coordinata da Milano da Musatti e sviluppata a Padova e Trieste dai suoi allievi Metelli e Kanizsa, e quella incarnata, sempre a Milano, da Agostino Gemelli. Mentre la scuola della Gestalt concepiva la percezione come il risultato di un'organizzazione dei dati sensoriali rispondente a principi o leggi autoctone, Gemelli si faceva promotore di un orientamento funzionalista, che condivideva l'approccio fenomenologico e anti-strutturalista della psicologia della Gestalt, ma al tempo stesso valorizzava gli aspetti non strettamente sensoriali della percezione, sottolineando il ruolo decisivo svolto dalla personalità nel costituirsi dell'atto percettivo, sia nei suoi aspetti stabili, intellettivi e caratterologici, sia in quelli temporanei, controllati da specifiche condizioni biologiche, psicologiche e sociali.

Alla base dell'orientamento di Gemelli stava infatti il concetto di personalità o individualità bio-psico-sociale, ovvero l'idea che le funzioni psichiche devono essere studiate nella loro organizzazione integrata e globale, che si struttura nel corso dello sviluppo come qualcosa di unico e irripetibile. In tal modo la ricerca si spostava, senza mai abbandonare le istanze poste dal metodo sperimentale, da un'indagine orientata sui processi a una centrata sull'uomo e la sua personalità, intesa come l'espressione di un'integrazione fra biologico, psichico e sociale, che si viene formando nel corso dello sviluppo, contrassegna in modo differenziato ciascun individuo e sta alla base di ogni processo mentale.

Inserendosi nel lavoro corale della Scuola di Gemelli, Cesa-Bianchi ha compiuto nella seconda metà degli anni ' 50 numerose ricerche sulla percezione e sul riconoscimento visivo, sui movimenti oculari, sulla stereoscopia, sul ruolo dei fattori affettivi nella percezione. In seguito, ma sempre in linea con gli orientamenti di fondo del suo maestro, egli si è venuto accostando al New Look di Jerome S. Bruner e Leo Postman che, accanto ai fattori autoctoni, ponevano in primo piano i fattori funzionali e personali: la percezione non è solo il risultato dell'organizzazione degli stimoli secondo determinate leggi, ma anche espressione delle condizioni del soggetto che interviene in modo attivo sul percetto a livello cognitivo e dinamico. 
La percezione diventa così per Cesa-Bianchi il risultato di molteplici fattori, sia autoctoni o strutturali, legati alle caratteristiche degli stimoli che provengono dall'ambiente esterno, sia personali e funzionali, che modulano i bisogni, le motivazioni, le aspettative, ma anche i meccanismi di difesa dei soggetti. A partire da qui, Cesa-Bianchi sottolinea con Witkin e Klein anche il ruolo svolto dagli stili di comportamento e di orientamento cognitivo nel determinare il risultato percettivo, e si accosta all'orientamento transazionale di Ames, Cantril e Ittelson, che considera l'adattamento non più nei soli termini di stimolo e risposta, ma come il risultato di un'interazione (transazione) tra stimoli ambientali e assunzioni inconsce precedentemente acquisite sulla natura degli stimoli, nel contesto di un' organizzazione totale del rapporto individuo-ambiente, e dunque in un'ottica decisamente ambientale. Alla fine degli anni Sessanta parte degli studi sulla percezione della scuola milanese di Marcello Cesa-Bianchi possono in effetti essere considerati tra i precursori della psicologia ambientale in Italia, con la formazione di ricercatori che nei decenni successivi si dedicheranno a tematiche psicologico-ambientali.

In tal modo, il problema della percezione in Cesa-Bianchi si è andato progressivamente allargando, o meglio si sono fatti sempre più sfumati $\mathrm{i}$ confini che lo separavano da altri ambiti psicologici, a partire da quello della personalità. La psicologia della percezione, e con essa l'intera psicologia generale, non si occupa più di processi intesi in senso astratto, ma è portata ad indagare anche le modalità secondo cui si svolge il funzionamento psichico del singolo individuo, in un'ottica differenziale. Di qui le indagini di Cesa-Bianchi sul funzionamento percettivo in soggetti normo-, sub- e non-vedenti, o collocati in diverse fasi del ciclo di vita, in particolare in età senile, dove la sempre minore efficienza degli apparati sensoriali nel corso del processo di invecchiamento può essere compensata o vicariata dall'utilizzo di strategie percettivo-cognitive che controllano la qualità e quantità di informazioni che il sistema visivo elabora, come la costanza percettiva, l'adattamento agli aspetti formali essenziali del percetto e il ricorso all'esperienza passata.

Cesa-Bianchi ha formato eccellenti percettologi. Tra i suoi primi allievi vanno segnalati Angelo Beretta e Riccardo Luccio, il primo purtroppo prematuramente scomparso, il secondo divenuto in seguito uno dei più autorevoli studiosi della scuola triestina di Kanizsa, con i quali Cesa-Bianchi ha scritto un volume di grande fortuna (Cesa-Bianchi et al., 1970) che ha costituito, e continua a rappresentare, un manuale fondamentale per ricchezza di informazioni, ampiezza di prospettive e integrazione di diversi orientamenti teorici. Attraverso l'esame di esperimenti, modelli teorici, 
delle polemiche del passato e del presente, il lettore è condotto nel cuore della psicologia della percezione e dei suoi problemi, almeno di quelli, ancora peraltro non del tutto risolti, aperti una trentina di anni fa.

Tra gli allievi percettologi della seconda generazione di Cesa-Bianchi un cenno merita Roberto Masini, scomparso prematuramente nel 1994 a soli 43 anni, che ha fornito fondamentali contributi anche nello studio delle immagini mentali, della soluzione dei problemi e della comprensione linguistica. Avvicinatosi anch'egli, tramite Kanisza, all'orientamento gestaltista, Masini ha svolto ricerche fondamentali sulla grandezza apparente di superfici e figure in funzione del loro completamento amodale, sulle superfici anomale e le loro condizioni di insorgenza, sui margini sfumati nella totalizzazione percettiva, e sul carattere intermodale di numerosi fenomeni percettivi, dalle illusioni ottiche ai fenomeni stereocinetici.

\section{La dimensione municipale della psicologia sperimentale a Milano}

Il 31 ottobre 1960 veniva inaugurata presso il Grattacielo Pirelli (che ora ospita il Consiglio Regionale lombardo) la nuova sede dell' Istituto di Psicologia Sperimentale del Comune di Milano (Cesa-Bianchi, 1960a). Le origini risalivano al 1907 come Gabinetto di Psicologia Sperimentale, ma deve essere sottolineato il cambio di denominazione in Laboratorio Civico di Psicologia pura e applicata avvenuto già nel 1908 (Redondi, 2008).

La prima direzione, affidata a Zaccaria Treves (1869-1911), fu di breve durata, interrotta dalla prematura morte, cosicché fu la lunga direzione di Casimiro Doniselli (1876-1960) (Butti, 2016), durata dal 1911 al 1951 (Cesa-Bianchi, 1960a), a caratterizzarne l'attività.

Le vicende belliche, con la distruzione completa della strumentazione, avevano reso la sede precaria. Essa era peregrinata dapprima in strutture dell'Università degli studi di Milano: da via Botticelli (presso l'Istituto di Fisiologia), all'Acquario Civico; da via Celoria (presso la Facoltà di Agraria) a via Vanvitelli (presso l'Istituto di Farmacologia). Infine aveva trovato sede presso un edificio scolastico in Via Morosini (Cesa-Bianchi, 1960a), ove aveva sede anche il Centro Medico Psicologico di Orientamento Scolastico e Professionale, diretto anch'esso da Marcello CesaBianchi. Quest'ultima struttura aveva ripreso l'attività dell' Istituto di Psicologia Sperimentale a partire dal 1952-1953, poiché nel 1950-1951, in coincidenza con la cessazione dell'attività di Casimiro Doniselli, era cessata di fatto anche l'attività dell' Istituto (Cesa-Bianchi, 1957a). 


\section{La psicologia dell'età evolutiva}

Con l'attivazione del Centro Medico Psicologico di Orientamento Scolastico e Professionale l'attività dell'Istituto di Psicologia riprendeva con una iniziativa settoriale, dedicata all'ambito dell'età evolutiva e indirizzata all'orientamento delle scelte scolastiche e dell'indirizzo professionale (stante anche la strutturazione allora vigente dell'ordinamento scolastico, che prevedeva al termine del ciclo di istruzione elementare la compresenza di differenti canali formativi).

Non si deve inoltre dimenticare l'esistenza di Scuole Speciali Comunali per minorati (per usare un termine d'epoca; oggi si parlerebbe di diversamente abili), al servizio delle quali l'Istituto di Psicologia Sperimentale del Comune di Milano (anche nella sua formulazione di Centro Medico Psicologico di Orientamento Scolastico e Professionale) si poneva come punto di riferimento (Istituto Italiano di Medicina Sociale, 1965). La storia di queste istituzioni scolastiche ${ }^{1}$ era andata di pari passo con quella delle strutture sanitarie e psicologiche municipali.

La produzione scientifica aveva condotto a una sperimentazione sugli interessi degli scolari della classe V elementare (Cesa-Bianchi, 1955) e in seguito a un ampliamento della stessa su tre livelli (V elementare, III media, V liceo scientifico) (Cesa-Bianchi e Perugia, 1955); non si deve infine dimenticare l'attività routinaria, che comportava l'esecuzione di circa 10.000 esami annuali (Cesa-Bianchi, 1963).

Negli anni Sessanta si affiancò a questa attività un altro filone di ricerca, sempre di pertinenza dell'età evolutiva, caratterizzato da una differente campionatura dei soggetti: non si trattava più degli scolari e alunni delle scuole di una grande città, ma degli allievi di una scuola professionale di una rilevante industria cartaria. Si trattava della Cartiera di Cairate, meglio nota come Cartiera Vita Mayer.

Negli anni Cinquanta del Novecento, sotto la guida di Astorre Mayer (1906-1977), esponente di spicco della Comunità ebraica milanese (Paganoni, 2010; Hamaui, 2016), erano stati istituiti alcuni servizi di grande rilievo, quali una Scuola Professionale e un Centro di Psicologia del Lavoro e Prevenzione Infortunistica, affidato a Marcello Cesa-Bianchi.

1 La Scuola speciale "Gaetano Negri” per Motulesi (dal 1992 confluita nell'I. C. Paolo e Larissa Pini) è del 1911; la Scuola speciale "Antonio Scarpa" (ora I. C. Antonio Scarpa) per Ambliopici [Minorati visivi] è del 1958; la Scuola speciale "Paolo Pini" per Disritmici [Epilettici], ora I. C. Paolo e Larissa Pini (già sezione staccata della Scuola speciale Treves De Sanctis dal 1947) si rese autonoma nel 1952 (Cesa-Bianchi e Sala, 1988); la Scuola speciale "Giulio Tarra" per Otologopatici è del 1919, insieme alla Scuola speciale per insufficienti mentali (Scuola speciale Treves De Sanctis per la cura medico-pedagogica dei fanciulli anormali psichici); la Scuola speciale per bambini gracili P. A. Giuliani (attivata nel 1922, si trasferirà nel Parco Ex-Trotter dal 1925). 
Fu promossa la pubblicazione di una collana di volumi, Studi e ricerche sui problemi umani del lavoro, che successivamente si tripartì in Studi e ricerche di psicologia; Studi e ricerche di psicologia del lavoro e della scuola; Esperienze e documentazioni sul lavoro e sulla scuola (con la pubblicazione di 56 fascicoli, fra il gennaio 1960 e il settembre 1964).

Ciò ci consente di inserire nella nostra analisi un secondo aspetto dell'attività di Marcello Cesa-Bianchi: quello relativo alla Psicologia del lavoro (Antonelli e Zocchi, 2013; Porro et al., 2017, 2019).

\section{La Psicologia del lavoro}

Oltre all'attività svolta in collaborazione con grandi imprese industriali, si deve ricordare che nella prima metà degli anni Cinquanta del secolo scorso a Milano la psicologia del lavoro poteva contare, nell'Università Cattolica del Sacro Cuore, su una possibilità operativa e formativa di rilievo. Si pensi che solo l'Università Cattolica del Sacro Cuore poteva rilasciare diplomi di specializzazione in Psicologia del lavoro e Psicotecnica a partire dal 1955.

Padre Agostino Gemelli (1878-1959) (Montanari, 2013; 2017) era impegnato anche a livello organizzativo e scientifico nazionale, quale Presidente della Commissione Nazionale per lo Studio e la Determinazione dei Profili Professionali, istituita nel 1953 dall'ENPI (Ente Nazionale per la Prevenzione degli Infortuni) sotto l'egida del Ministero del Lavoro e della Previdenza Sociale (Spaltro, 2003). Gemelli tenne la presidenza della Commissione fino al 1957.

Cesa-Bianchi, allievo diretto di padre Gemelli (Vito et al., 1960), riuscì a inserire la Psicologia nell'ambito della Facoltà di Medicina e Chirurgia dell'Università degli Studi di Milano, impresa che non era riuscita, nonostante strenui e notevoli tentativi, a Cesare Musatti (1897-1989), incardinato nella Facoltà di Lettere e Filosofia della stessa Università. Ciò accadde in Clinica del Lavoro "Luigi Devoto", nella quale erano state attivate una serie di Sezioni, che si avvalevano della collaborazione di grandi industrie (come la Montecatini).

Per tornare all'ambito industriale più sopra citato, era stata attivata una Sezione di ricerche di medicina e igiene del lavoro nelle industrie grafiche, cartotecniche, trasformatrici della carta e affini della Provincia di Milano.

Un altro ambito di ricerca era quello delle industrie tessili: anche in questo campo era stata attivata una Sezione per lo studio del Fattore Umano nelle Industrie Tessili, nella quale si espletava l'attività di Marcello Cesa-Bianchi e dei suoi collaboratori (anche con la pubblicazione di partico- 
lari sussidi editoriali, la cui analisi è attualmente in corso, in collaborazione con la cattedra di Storia della Medicina dell'Università degli Studi di Milano).

La prima sede della cattedra di Psicologia della Facoltà di Medicina e Chirurgia dell'Università degli Studi di Milano fu proprio in via San Barnaba 8, presso la Clinica del Lavoro milanese, in un locale poco discosto dall'atrio d'ingresso: questo dato è stato sottostimato dalla storiografia medica e psicologica milanese, che ha sempre privilegiato il collegamento con la realtà Municipale.

Si venivano così assommando in Cesa-Bianchi tutte le caratteristiche che lo avrebbero reso uno dei protagonisti della psicologia medica del tempo (e per molto tempo ancora): la compresenza delle competenze mediche, psicologiche e pedagogiche; la direzione di un'Istituzione municipale; l'attività di rilievo in due Università cittadine (infatti egli manteneva anche la sua attività presso l'Università Cattolica del Sacro Cuore); per quanto poi riguarda la psicologia del lavoro, non si doveva dimenticare quella prestata in industrie di primo rilievo nazionale e internazionale.

Nell'agosto 1957 una breve nota dedicata a La psicologia applicata nella medicina del lavoro (Cesa-Bianchi, 1957b) preannunciava, in una sorta di manifesto programmatico, il lavoro futuro.

La qualifica di Cesa-Bianchi come Dirigente la Sezione di Psicologia industriale presso la Clinica del Lavoro ci indica la formalizzazione della Sezione stessa, ma è l'analisi del testo a renderci una vivida immagine delle concezioni di Cesa-Bianchi in tema di psicologia del lavoro.

Innanzitutto, l'esordio costituisce una rivendicazione della visione personalistica propria della scuola gemelliana: l'oggetto dell'attività è l'uomo considerato in tutte le sue dimensioni - biologiche, psicologiche e anche sociologiche (Cesa-Bianchi, 1957b).

In questo contesto, e anche per rispondere ad un ritardo italiano nelle applicazioni di psicologia del lavoro, si situava la costituzione di un Servizio Medico-Psicologico nel contesto della Clinica del Lavoro milanese. "Tale Servizio ha lo scopo di studiare da un punto di vista psicologico, cioè dal punto di vista della soggettività di ciascuno, le diverse manifestazioni del comportamento dell'uomo, sia che esse si riferiscano al lavoro, sia che riguardino altri aspetti dell'agire e del pensare umano. E proprio in questo punto di vista soggettivo sta l'essenza dell'impostazione psicologica: che un ambiente sia o no luminoso, rumoroso o umido, penserà il fisico o l'ingegnere a stabilirlo; che un ambiente sia o no dannoso alla salute, penserà il medico a determinarlo; ma che un ambiente venga percepito da un determinato individuo come luminoso o rumoroso o umido o dannoso 
deve essere lo psicologo a studiarlo, ad indagarne i fattori causali e le modalità, a prevederne le conseguenze, a suggerirne le terapie" (Cesa-Bianchi, 1957b, p. 48).

Si trattava di un programma ambizioso. Le vicissitudini della sede della cattedra di Psicologia nella Facoltà di Medicina e Chirurgia dell'Università degli Studi di Milano possono aiutarci a comprendere la complessità della situazione.

All'atto dell'inaugurazione della nuova sede dell' Istituto di Psicologia Sperimentale del Comune di Milano, dopo un periodo di attivazione e di presenza dell'attività psicologica presso la Clinica del Lavoro degli Istituti Clinici di Perfezionamento, nel 1960-1961, la cattedra di Psicologia Sperimentale, coperta da Marcello Cesa-Bianchi per incarico, risultava ospite della Clinica delle Malattie Nervose e Mentali presso il Padiglione Ponti dell'Ospedale Maggiore Policlinico, al tempo diretta da Ermenegildo Gastaldi (1907-1973) (Bock Berti, 1999; Gastaldi et al., 2012).

Nel 1961-1962 la cattedra di Psicologia risultava ufficialmente incardinata nell'Istituto di Medicina del Lavoro presso gli Istituti Clinici di Perfezionamento.

Dal 1962-1963 la cattedra di Psicologia risultava come Cattedra non raggruppata in Istituto. L'indirizzo di via Fabio Filzi 20 ci indica che la sede si era definitivamente trasferita l'Istituto di Psicologia Sperimentale del Comune di Milano: essa sarebbe rimasta per oltre un quarantennio ospite di strutture comunali (dapprima in Via Francesco Sforza 23; indi in via Tommaso Pini 1), confermando che la duplice funzione, universitaria e municipale, di Marcello Cesa-Bianchi aveva saputo dare forza e stabilità alla presenza della psicologia nel mondo medico e cittadino.

Evoluzione naturale era stato l'inserimento della Psicologia medica nei corsi della Facoltà di Medicina e Chirurgia e l'istituzione del relativo posto di ruolo, assegnato per concorso a Marcello Cesa-Bianchi.

In quegli anni si veniva delineando anche un ruolo internazionale per Marcello Cesa-Bianchi e i suoi collaboratori, grazie anche alla partecipazione a ricerche promosse dalla CECA (Comunità Europea del Carbone e dell'Acciaio) nel periodo della sua autonomia (fino al 1967) e nel successivo alveo della Commissione delle Comunità europee.

Ciò consentì di affrontare rilevanti temi di psicologia applicata, con attenzione precipua alla sicurezza (Cesa-Bianchi, 1967; 1972; Cesa-Bianchi e Mantovani, 1974a, 1976), tema di grande importanza e attualità ancor oggi. 


\section{I cambiamenti degli anni Settanta}

Gli ultimi anni Sessanta e gli anni Settanta furono forieri di cambiamenti profondissimi nella compagine sociale italiana e anche l'ambito della psicologia (e della psicologia medica) non ne rimase estraneo.

Prima di affrontare un altro tema di grande rilevanza (la formazione psicologica del medico, allargabile anche al più generale ruolo e all' organizzazione della psicologia), possiamo fornire alcune puntualizzazioni su come i campi d'attività di Marcello Cesa-Bianchi fin qui analizzati siano stati in seguito sviluppati.

L'impegno nelle istituzioni comunali proseguì, come anche l'attività di orientamento e screening. Andava invece mutando la struttura delle Scuole speciali: le loro competenze non erano state dismesse, ma progressivamente si andò delineando l'inserimento degli allievi portatori di disabilità nel contesto scolastico generale. Emerse così la necessità di sussidiarietà da parte delle associazioni del volontariato: in questo contesto era imprescindibile una rigorosa formazione psicologica.

Valga l'esempio dell'Associazione Paolo Pini, presieduta per un cinquantennio da Marcello Cesa-Bianchi e tuttora attiva nel panorama del Terzo Settore milanese: da struttura di collegamento con la Scuola speciale "Paolo Pini" per Disritmici [Epilettici], essa allargò il suo spettro di attività al supporto alle famiglie con componenti portatori di disabilità gravi.

Anche l'evoluzione della Psicologia del lavoro seguì il mutare del contesto generale: la psicotecnica e la questione dei profili professionali furono affiancati, come già ricordato, dai temi della sicurezza, dell'ergonomia, dell'invecchiamento della popolazione lavorativa.

\section{La Psicologia medica}

Proporre una riflessione sulla Psicologia medica verso la fine degli anni Cinquanta significava da un lato confrontarsi con la storia della psicologia in senso generale, e dall'altro tenere conto dell'attualità (anche nei suoi aspetti controversi).

In un testo del 1957 (Cesa-Bianchi, 1957c) Marcello Cesa-Bianchi ripercorre le tappe evolutive delle discipline psicologiche, circoscrivere il campo della Psicologia medica. Egli prende come punti di riferimento due opere al tempo ritenute classiche: quella di Emil Kretschmer (1888-1964) (Kretschmer, 1952) e quella di Gregory Zilboorg (1890-1959) e George W. Henry (1889-1964) (Zilboorg e Henry, 1941²).

2 Si deve segnalare che le traduzioni in lingua italiana sono comparse con il titolo, fuorviante, di Storia della psichiatria. 
Marcello Cesa-Bianchi analizza, con riferimenti specifici, la psicologia fisiologica, la dottrina delle localizzazioni cerebrali e le reazioni antilocalizzatorie $^{3}$, il behaviorismo e la dottrina dei riflessi condizionati, la psicoanalisi ${ }^{4}$, la scuola personalistica, le scuole caratterologiche ${ }^{5}$, la psicologia clinica. A proposito di quest'ultima, egli riferisce dei due principali modi di intenderla: da un lato come psicologia applicata allo studio del malato di mente, dall'altro come psicologia che utilizza il metodo clinico, inteso quest'ultimo come superamento e integrazione del metodo analitico e del metodo sincretico (Cesa-Bianchi, 1957c). La figura dello psicologo clinico emerge come quella di un medico ben diverso dallo psichiatra e la psicologia clinica viene da Cesa-Bianchi inserita nel contesto della psicologia applicata.

La psicologia medica viene così quasi a sovrapporsi con la psicologia tout-court, anche perché, se la psicologia non può semplicisticamente considerarsi una branca della medicina, i problemi psicologici che interessano il medico vanno ben oltre quelli circoscritti alla diagnosi e alla terapia dei disturbi dell'attività psichica (Cesa-Bianchi, 1957c).

Marcello Cesa-Bianchi riconfermò di lì a pochi anni queste sue posizioni, ma deve essere evidenziato il corollario che dai due lavori citati emerge: quello della necessità dell'inserimento della Psicologia medica come insegnamento ufficiale sia nel Corso di Laurea in Medicina e Chirurgia (Cesa-Bianchi, 1957c), sia nella formazione post-laurea delle Scuole di specializzazione (fatto questo che si era iniziato a verificare proprio grazie al suo impegno) (Cesa-Bianchi, 1962).

3 Egli collega l'apparentemente contraddittoria coesistenza delle due opposte posizioni ad un errore di confusione fra la localizzazione della lesione e la localizzazione della funzione.

4 Con la citazione delle classiche posizioni di Sigmund Freud (1856-1939), Alfred Adler (1870-1937), Carl Gustav Jung (1875-1961), Melanie (Reizes) Klein (1882-1960), Karen (Danielsen) Horney (1885-1952) e Anna Freud (1895-1982). A riguardo delle evoluzioni del tempo, Cesa-Bianchi cita la medicina psicosomatica di Franz Gabriel (Ferenc Gàbor) Alexander (1891-1964), la terapia centrata sul cliente di Carl Ramson Rogers (1902-1987), l'impostazione di Josef Remi Nuttin (1909-1988), l'antropoanalisi di Ludwig Binswanger (1881-1966), la logoterapia di Viktor Emil Frankl (19051997).

5 Basate su criteri strettamente naturalistici, come quelle di Kretschmer o di William Herbert Sheldon (1898-1977); basate su criteri prevalentemente psicologici, come quelle di Gerardus Heymans (1857-1930), René Le Senne (1882-1954), Jung, Eric Rudolf Jaensch (1883-1940) e Gerhard Pfahler (1897-1976); basate su criteri psicologici, come quelle di Ludwig Klages (1872-1956), Eduard Franz Ernst Spranger (1882-1963) e Philipp Lersch (1898-1962). Si noti come alcune delle posizioni citate fossero state compromesse (Pfahler) o ambivalenti (Spranger) con il regime nazista. 


\section{La Psicologia medica nella formazione del medico}

Nel 1959 fu pubblicata un'attenta analisi dei problemi collegati alla selezione e al controllo e orientamento degli studenti di medicina (Bestetti e Lucchelli, 1959), e il lavoro aveva vinto il Premio Roche del 1958.

La presenza della psicologia, nella pur attenta analisi degli autori, era pressoché inesistente (essendo citato il Medical College Admission Test MCAT -, senza però connotarlo nella sua dimensione psicologica), così come lo era di fatto nelle Facoltà di Medicina e Chirurgia delle Università italiane.

Il 4 agosto 1958 era stato pubblicato il decreto che istituiva la Facoltà di Medicina e Chirurgia dell'Università Cattolica del Sacro Cuore e all'articolo 21 veniva introdotta una grande novità: per l'iscrizione al corso di laurea in medicina e chirurgia gli aspiranti dovranno essere sottoposti ad un esame medico e ad un esame attitudinale e psicodiagnostico: esami che hanno lo scopo di constatare se gli aspiranti hanno le qualità fisiche e attitudinali per poter attendere agli studi superiori di medicina (CesaBianchi et al., 1959).

Gli stessi dati di Bestetti e Lucchelli dimostravano in realtà una presenza diffusa dell'applicazione dei reattivi mentali (per usare un termine d'epoca) nella selezione degli studenti. Cesa-Bianchi, Spaltro e Trentini eseguirono una prima sperimentazione applicativa del MCAT e del Minnesota Multiphasic Personality Inventory (MMPI) sopra un campione di studenti delle scuole superiori milanesi: si trattava di una ricerca pionieristica per il nostro paese.

L'attenzione doveva essere posta anche agli studenti durante il loro cursus studiorum. Dal 15 marzo 1964 fu attivato un Consultorio Medico Psicologico interuniversitario (fra l'Università degli Studi e il Politecnico di Milano) (Cesa-Bianchi, 1966b) e contemporaneamente vennero indagate le abitudini di studio degli studenti universitari, nell'ambito di una collaborazione fra psicologi medici e igienisti (Giovanardi et al., 1965). I dati riguardanti gli studenti della Facoltà di Medicina e Chirurgia furono pubblicati solo nel 1972 (Giovanardi et al., 1972), quando erano già intervenute le modifiche legislative che avevano eliminato le restrizioni all'accesso universitario (all'epoca della pubblicazione il lavoro aveva, dunque, anche un valore per l'analisi storiografica).

La necessità di una formazione psicologica del medico e dello specialista doveva andare di pari passo con la presenza strutturata della psicologia nelle facoltà mediche universitarie (Cesa-Bianchi, 1969; Cesa-Bianchi e Beretta, 1969). Marcello Cesa-Bianchi aveva affrontato sin dai primi anni Sessanta il problema della formazione psicologica degli specialisti (CesaBianchi 1960b; 1965b). 
Si andava nel frattempo ipotizzando una presenza autonoma disciplinare per la psicologia nelle Università italiane (Cesa-Bianchi e Musatti, 1967).

Gli anni Settanta avrebbero posto la psicologia medica di fronte a problemi e prospettive nuove, identificate con preveggenza nello sviluppo della dimensione dipartimentale (Cesa-Bianchi, 1974).

A conclusione di questo contributo crediamo utile ricordare gli apporti autobiografici (Cesa-Bianchi et al., 2009; Mancino, 2012; Cesa-Bianchi, 2013 ), che valgono a definire, congiuntamente a quelli storiografici (Cristini e Porro, 2017; Cipolli et al., 2018; Cesa-Bianchi et al., 2018; Cristini et al., 2019; Cristini e Porro, 2019), la figura e l'opera di Marcello CesaBianchi.

\section{Riferimenti bibliografici}

Antonelli, M., \& Zocchi, P. (Eds.) (2013). Psicologi in fabbrica. Storie e fonti. Roma: Aracne.

Bestetti, A., \& Lucchelli, P. (1959). Avviamento alla professione medica. Selezio$n e$, controllo ed orientamento degli studenti di medicina. Milano: Il Giardino di Esculapio.

Bock Berti, G. (1999). Gastaldi Ermenegildo (Gildo). In Dizionario Biografico degli Italiani, Vol. 52. Roma: Istituto della Enciclopedia Italiana.

Butti, N. (2016). Casimiro Doniselli e l'Istituto civico di psicologia: una storia milanese quasi dimenticata. Storia in Lombardia, 36(1), 25-56.

Cavagna, D., Cristini, C., \& Cesa-Bianchi, M. (2017). Rassegna storica degli indirizzi quantitativi e qualitativi in psicologia. Ricerche di Psicologia, 40(1), 25-66.

Cesa-Bianchi, G., Porro, A., Cristini, L., \& Cristini, C. (2018). Misurare i vecchi: Il contributo di Agostino Gemelli e Marcello Cesa-Bianchi. In Atti Congressuali. $63^{\circ}$ Congresso Nazionale SIGG (276-277). Pisa: Pacini.

Cesa-Bianchi, M. (1951). L'applicazione dei reattivi mentali nello studio dell'invecchiamento. Archivio di Psicologia, Neurologia e Psichiatria, 12(4-5), 390393.

Cesa-Bianchi, M., \& Perugia, A. (1952a). Metodi statistici in psicologia. Firenze: Editrice Universitaria.

Cesa-Bianchi, M., \& Perugia, A. (1952b). Adattamento italiano del reattivo Wechsler-Bellevue per la valutazione dell'intelligenza (con 32 tabelle e una figura). In Contributi del Laboratorio di Psicologia (Vol. XLI, 56). Milano: Vita e Pensiero.

Cesa-Bianchi, M. (1955). Contributo allo studio degli interessi a livello della V elementare (con 6 tabelle). In Contributi del Laboratorio di Psicologia, XLVIII. Milano: Vita e Pensiero. 
Cesa-Bianchi, M., \& Perugia A. (1955). Ricerca sulla corrispondenza fra rendimento ed interessi tecnici a tre livelli dell'età evolutiva (con 9 tabelle e 6 grafici). In Contributi del Laboratorio di Psicologia, (Vol. XLVIII, 21-40). Milano: Vita e Pensiero.

Cesa-Bianchi, M. (1957a). Centro Medico-psicologico di Orientamento Scolastico e Professionale. Milano, Comune di Milano.

Cesa-Bianchi, M. (1957b). La psicologia applicata nella medicina del lavoro. Attualità ospedaliera, 2(4), 47-48.

Cesa-Bianchi, M. (1957c). La psicologia medica. Minerva Medica, 48(100), 4243-4247.

Cesa-Bianchi, M., Spaltro, E., \& Trentini, G. (1959). Criteri orientativi per gli studi medici. In Contributi dell'Istituto di Psicologia (Vol. LXVIII, 102-118). Milano: Vita e Pensiero.

Cesa-Bianchi, M. (1960a). Istituto di psicologia sperimentale del Comune di Milano. L'Organizzazione Sanitaria, 5, 5, 6-12.

Cesa-Bianchi, M. (1960b). Psicologia e medicina. Gazzetta Sanitaria, 9, 9, 431433.

Cesa-Bianchi, M. (1962). Problemi e prospettive della psicologia medica. Gazzetta Sanitaria, 11, 9, 3-8.

Cesa-Bianchi, M. (1963). Operosità scientifica e carriera didattica. Milano, Tipografia Memo.

Cesa-Bianchi, M. (1965a). L'impostazione delle ricerche psicologiche sull'invecchiamento. In M. Perez (Ed.), Psicologia e psicopatologia dell'età senile. Atti del V Convegno medico-sociale dell'O.N.P.I., ( 197-209). Roma, Il Pensiero Scientifico.

Cesa-Bianchi, M. (1965b). La formazione psicologica del pediatra. In Pediatria biologica e psicologica. Atti del Symposium sui rapporti fra psicologia e pediatria tenuto alla Facoltà di Medicina e Chirurgia dell'Università Cattolica del Sacro Cuore. Roma 3-6 novembre 1962 (298-302). Milano: Vita e Pensiero.

Cesa-Bianchi, M. (1966a). Il problema metodologico nella psicologia attuale. $M i$ nerva Medica, 57(28), 1233-1239.

Cesa-Bianchi, M. (1966b). Osservazioni preliminari tratte dall'attività di un consultorio psicologico per universitari. In Atti IV del Convegno Nazionale e della I Conferenza Europea di Servizio Sanitario Universitario (pp. 347-351). Roma: Quintilly.

Cesa-Bianchi, M. (1967). Ricerca nell'industria siderurgica italiana. Lussemburgo, Comunità Europea del Carbone e dell'Acciaio.

Cesa-Bianchi, M., \& Musatti, C. (Eds.) (1967). La psicologia nell'Università e nella società di oggi e di domani. In Le scienze dell'uomo e la riforma universitaria. Studi e ricerche condotti dal Centro nazionale di prevenzione e difesa sociale (335-413). Roma-Bari: Laterza.

Cesa-Bianchi, M. (1969). La formazione psicologica del medico. Federazione Medica, 22(10), 3-7.

Cesa-Bianchi, M., \& Beretta, A (1969). Psicologia. In V. Ghetti (Ed.), Guida CIBA per il giovane medico. Milano: CIBA. 
Cesa-Bianchi, M., Beretta, A., \& Luccio, R. (1970). La percezione. Un'introduzione alla psicologia della visione. Milano: FrancoAngeli.

Cesa-Bianchi, M. (1972). Affidabilità e sicurezza. Lussemburgo, Commissione delle Comunità Europee, Direzione generale Affari Sociali, Ufficio delle pubblicazioni ufficiali delle Comunità Europee.

Cesa-Bianchi, M. (1974). L'insegnamento della psicologia nella Facoltà di Medicina: problemi e prospettive. In Formazione psicologica del medico. Atti del Quarto Congresso Nazionale della Società Italiana di Medicina Psicosomatica. Messina 31 maggio-3 giugno 1973 (39-49). Roma: SEU.

Cesa-Bianchi, M., \& Mantovani, G. (1974). Igiene e sicurezza del lavoro. Lussemburgo: Commissione delle Comunità Europee.

Cesa-Bianchi, M., \& Mantovani, G. (1976). Training in industrial safety. Lussemburgo: Commissione delle Comunità Europee.

Cesa-Bianchi, M. (1987). Definizione, indirizzi e metodi della psicologia. In G. Gulotta (Ed.), Trattato di psicologia giudiziaria nel sistema penale ( 29-72). Milano: Giuffrè.

Cesa-Bianchi, M., \& Sala, G. (1988). Umanità e scienza in medicina. Milano,: FrancoAngeli.

Cesa-Bianchi, M. (1998). Giovani per sempre? L'arte di invecchiare. Roma-Bari: Laterza.

Cesa-Bianchi, M., Porro, A., \& Cristini, C. (2009). Sulle tracce della psicologia italiana. Storia e autobiografia. Milano: Franco-Angeli.

Cesa-Bianchi, M. (2013). La formazione di un giovane psicologo alla scuola di Gemelli. Ricerche di Psicologia, 36(4), 411-415.

Cipolli, C., Cristini, C., \& Porro, A. (2018). Ricordando Marcello Cesa-Bianchi. Ricerche di Psicologia, 41(1), 9-11. DOI 10.3280/RIP2018-001-001

Cristini, C., Cesa-Bianchi, M., Cesa-Bianchi, G., \& Porro, A. (2011). L'ultima creatività. Luci nella vecchiaia. Milano: Springer Verlag.

Cristini, C., \& Porro, A. (2017). Per un'ergobiografia di Marcello Cesa-Bianchi. Ricerche di Psicologia, 40(4), 443-528. DOI 10.3280/RIP2017-004-004.

Cristini, C., Cesa-Bianchi, G., Cristini, L., \& Porro, A. (2019). Doctor-patient relationship: The work of Marcello Cesa-Bianchi. Mediterranean Journal of Clinical Psychology, 7, (suppl. 2), 95-96. DOI: 10.6092/22821619/2019.7.2267.

Cristini, C., \& Porro, A. (2019). Marcello Cesa-Bianchi e l'Istituto di Psicologia della Facoltà Medica dell'Università di Milano: la rinascita della psicologia italiana. In S. Di Nuovo, M. Sinatra \& T. Vecchi (Eds.), Ricordare il passato per costruire il futuro: la memoria storica della psicologia ( 13-23). Lecce, Pensa MultiMedia.

Gastaldi, C., Gastaldi, M., \& Lia, L. (2012). Perché resistemmo perché resistiamo. Vita e impegno civile di Gildo Gastaldi. S.1., Àncora arti grafiche.

Giovanardi, A., Cesa-Bianchi, M., Saccani, C. F., Fabbrichesi, A. M., Criscuolo, A., Magistretti, G., Marangoni, L., Pimpinelli, E., Autelitano, G., Rossi, G., \& Capricci, M. (1965). Inchiesta sulle abitudini di studio negli universitari di Milano. Rivista Italiana di Medicina e Igiene della Scuola, 11(1), 9-14. 
Giovanardi, A., Cesa-Bianchi, M., Autelitano, G., \& Scalera, L. (1972). Indagine sulle attitudini di studio degli studenti della Facoltà di Medicina. Difesa Sociale, 51(2), 44.

Hamaui, R. (2016). Ebrei a Milano. Due secoli di storia fra integrazione e discriminazioni. Bologna: il Mulino.

Istituto Italiano di Medicina Sociale (1965). I centri sanitari delle scuole speciali del comune di Milano. Roma: Istituto Italiano di Medicina Sociale.

Kretschmer, E. (1952). Manuale teorico pratico di psicologia medica. Firenze: Sansoni.

Mancino, E. (2012). Sempre in anticipo sul mio futuro. Autobiografia di Marcello Cesa-Bianchi. Napoli: Guida.

Meehl, P. E. (1954). Clinical versus statistical prediction. A theoretical analysis and a review of the evidence. Minneapolis: University of Minnesota Press.

Montanari, I. (2013). Agostino Gemelli e la psicotecnica nel secondo dopoguerra. Ricerca, applicazione e divulgazione nelle carte del Fondo Gemelli-Scolari. In M. Antonelli \&, P. Zocchi (Eds.), Psicologi in fabbrica. Storie e fonti (pp 129-153). Roma: Aracne.

Montanari, I. (2017). Agostino Gemelli psicologo. Una ricostruzione storiografi$c a$. Milano: EDUCatt.

Paganoni, M. (Ed.) (2010). Per ricostruire e ricostruirsi. Astorre Mayer e la rinascita ebraica tra Italia e Israele. Milano: FrancoAngeli.

Poli, E. (1965). Metodologia medica. Principi di logica e pratica clinica. Milano: Rizzoli.

Porro, A., Cristini, C., Galimberti, P. M., Falconi, B., Lorusso, L., \& Franchini, A. F. (2017). A Milano, sessant'anni fa: medicina del lavoro e psicogerontologia. Giornale Italiano di Medicina del Lavoro ed Ergonomia, 39, (suppl. 3), 66.

Porro, A., Falconi, B., Lorusso, L., Galimberti, P. M., Riva, A. M., Franchini, A. F., \& Cristini, C. (2019). Medicina del Lavoro e Psicologia del Lavoro: un incontro nella Milano del secondo dopoguerra. La Medicina del Lavoro, 110(1), 63-74.

Redondi, P. (2008). Educare per la vita. L'Istituto Civico di Psicologia Sperimentale. In E. Canadelli \& P. Zocchi, (Eds.), Milano scientifica 1875-1924. La rete del grande Politecnico (Vol. 1, 277-301). Milano: Sironi Editore.

Spaltro E. (2003). La forza di fare le cose. Fondamenti di psicologia del lavoro. Bologna: Pendragon.

Vito, F., Alberini, F., Quadrio, A., Spaltro, E., Ancona, L., Cesa-Bianchi, M., Iacono, G., \& Galli, F. (1960). Padre Gemelli e i problemi del lavoro. Milano: Vita e Pensiero.

Zilboorg, G., \& Henry, G. W. (1941). A History of medical psychology. New York: Norton. 